\title{
Ortorexia nervosa: reflexões sobre um novo conceito
}

\author{
Orthorexia nervosa: reflections \\ about a new concept
}

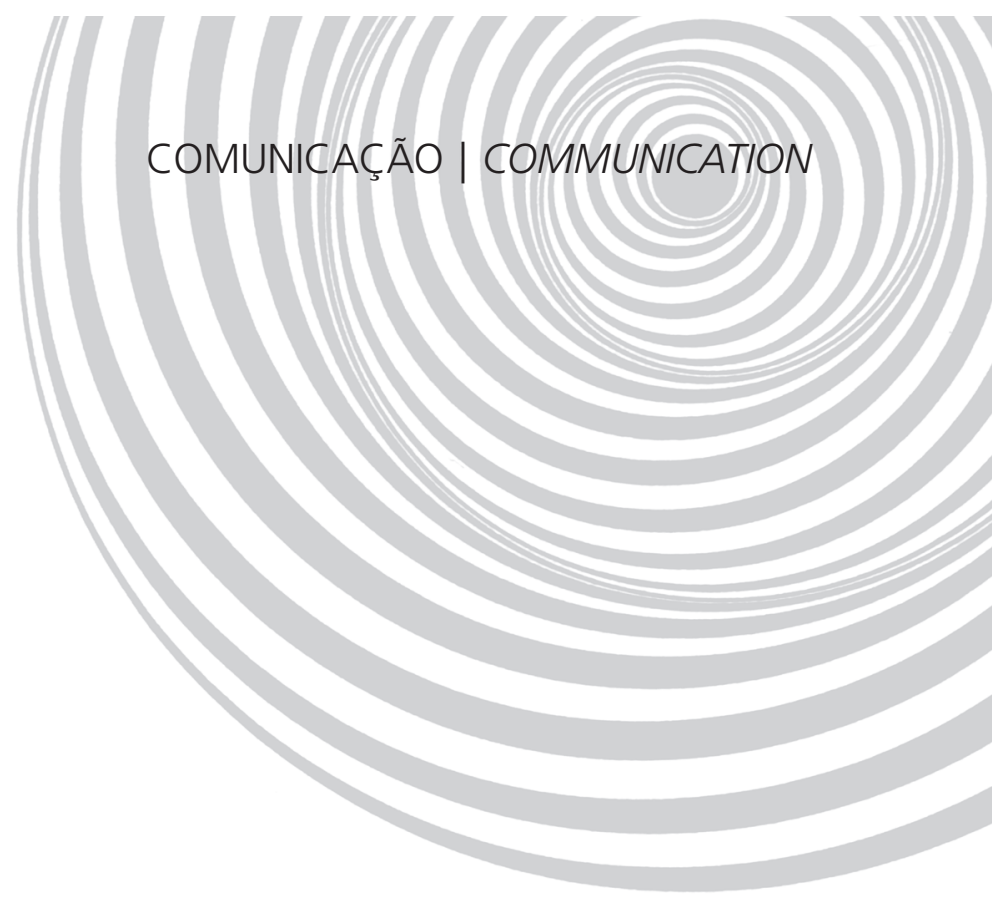

Márcia Cristina Teixeira MARTINS 1

Marle dos Santos ALVARENGA²

Sílvia Viviane Alves VARGAS ${ }^{1}$

Karen Sayuri Cabral de Jesus SATO'

Fernanda Baeza SCAGLIUSI ${ }^{3}$

RE S U M O

Ortorexia nervosa é o termo descrito para o comportamento obsessivo patológico caracterizado pela fixação por saúde alimentar. O quadro ainda não foi oficialmente reconhecido como um transtorno alimentar, mas discute-se o conceito, suas características, interações e sintomas. No presente trabalho foi realizada uma revisão dos vinte e um artigos publicados desde 1997, quando o comportamento da ortorexia nervosa foi inicialmente descrito. Foram apontadas semelhanças e diferenças entre o comportamento alimentar observado na ortorexia nervosa e nos transtornos alimentares mais frequentes (anorexia e bulimia nervosa). Um instrumento foi desenvolvido e validado para detecção do quadro ortoréxico. Os estudos apontam alguns grupos vulneráveis à ortorexia nervosa: estudantes de medicina, médicos, nutricionistas, pessoas com sintomas de ansiedade, obsessivocompulsivos e aqueles que supervalorizam o corpo perfeito. A ortorexia nervosa é situada a partir de uma análise dos conceitos de atitude alimentar e alimentação saudável, procurando um foco biopsicossocial para a alimentação adequada e não apenas um foco fisiológico. Não existem estudos investigativos sobre a ortorexia nervosa no Brasil, mas o tema deve ser discutido para alertar os profissionais da área da saúde sobre a existência desse comportamento inadequado e suas possíveis consequências não só para a saúde física e emocional, mas também para a visão de alimentação saudável.

Termos de indexação: Comportamento alimentar. Hábitos alimentares. Nutrição humana. Ortorexia nervosa. Transtornos da alimentação.

A B S T R A C T

Orthorexia nervosa is a new term described as an obsessive pathological behavior characterized by fixation on healthy eating. It has not yet been officially recognized as an eating disorder, but its concept, characteristics,

\footnotetext{
1 Centro Universitário Adventista de São Paulo, Faculdade de Nutrição. Estrada de Itapecerica, 5859, 05858-001, São Paulo, SP,

Brasil. Correspondência para/Correspondence to: M.C.T. MARTINS. E-mail: <marciactm@yahoo.com.br>.

2 Universidade de São Paulo, Faculdade de Saúde Pública, Departamento de Nutrição. São Paulo, SP, Brasil.

3 Universidade Federal de São Paulo, Departamento de Ciências da Saúde, Campus Baixada Santista. Santos, SP, Brasil.
} 
346 | M.C.T. MARTINS et al.

interactions and symptoms have been discussed. This work presents a review of the articles published on the theme since 1997, when orthorexic behavior was first described. Similarities and differences between orthorexic behavior and the more common eating disorders, such as anorexia nervosa and bulimia nervosa, are discussed. To date, one instrument was developed and validated to detect orthorexic behavior. Studies indicate that some groups are more vulnerable to orthorexia nervosa: medical students, physicians, dietitians, individuals with anxiety symptoms, obsessive-compulsive individuals and those who overvalue a perfect body. The discussion on orthorexia nervosa is based on analyzing the concepts of eating attitude and healthy eating, and seeks a biopsychosocial focus for proper eating in addition to a physiological focus. To date, there are no studies about orthorexia nervosa in Brazil. Nevertheless, this subject is worthy of discussion since health professionals should be made aware of the existence of this deviant behavior and its possible consequences, not only for someone's physical and emotional health, but also for the understanding of healthy eating.

Indexing terms: Feeding. Food habits. Human nutrition. Orthorexia nervosa. Eating disorders.

\section{N T R O D U ÇÃ O}

A crescente preocupação com uma vida mais saudável, aliada ao conhecimento dos muitos fatores que afetam a saúde humana (genéticos, ambientais, comportamentais, culturais, dietéticos, entre outros), tem gerado também maior interesse pelo alcance de uma alimentação saudável' . A ideia de que a dieta exerce um importante papel na promoção da saúde e prevenção de doenças está cada vez mais presente na consciência coletiva ${ }^{2}$. No entanto, o foco de discussão sobre a "alimentação saudável" é usualmente apenas o biológico, e a alimentação adequada é pensada em função de uma dieta que atenda às recomendações nutricionais. Deve-se considerar que um foco apenas biológico pode ser perfeccionista e, consequentemente, não realista ${ }^{3}$. A alimentação saudável não deve envolver restrições. Não se recomenda a classificação dos alimentos em "bons" e "ruins" ou em "saudáveis" e "não saudáveis". Uma visão biopsicossocial sobre a alimentação saudável implica também que o julgamento sobre o que é saudável ou não depende de muitos fatores, tais como: história individual e familiar, cultura, religião, aspectos econômicos, experiência pessoal, preferências e aversões, conhecimentos e crenças, entre outros ${ }^{4,5}$.

Os Transtornos Alimentares (TA) são graves distúrbios psiquiátricos considerados importantes problemas de saúde. Os quadros clássicos têm seus critérios diagnósticos definidos pela Organização Mundial de Saúde (OMS) ou Associação Americana de Psiquiatria (APA), enquanto outros quadros considerados não oficiais têm sido sugeridos nos últimos anos, denominados muitas vezes de comer transtornada (do inglês, disordered eating) ${ }^{6}$. Um quadro recentemente apresentado é denominado pelo neologismo (do grego, orthos significa correto e orexis, apetite) Ortorexia Nervosa (ON). Descrita como um comportamento obsessivo patológico, a ortorexia nervosa caracteriza-se pela fixação por saúde alimentar, qualidade dos alimentos e pureza da dieta. Tal comportamento alimentar é ainda pouco explorado na literatura científica por diversas razões. É possível que, por não ser um TA oficialmente reconhecido ${ }^{7}$, a ortorexia nervosa tenha número limitado de trabalhos publicados. Há que se considerar, ainda, a delicadeza desse assunto claramente paradoxal, por focalizar o lado insalubre de um comportamento alimentar "obsessivamente saudável". A compreensão e a abordagem equilibrada desse tema podem ser particularmente difíceis e até mesmo de menor interesse para profissionais da área de alimentação e saúde, constantemente centrados em propagar a adoção de hábitos alimentares saudáveis, principalmente do ponto de vista biológico.

A temática da ortorexia nervosa ainda não foi explorada na literatura científica nacional. Para suprir essa lacuna, o presente trabalho teve como objetivo descrever o comportamento ortoréxico a partir das discussões fenomenológicas e dos dados epidemiológicos disponíveis nos trabalhos publicados até o momento. O comportamento ortoréxico foi comparado aos comportamentos alimentares característicos dos TA mais frequen- 
tes - Anorexia Nervosa (AN) e Bulimia Nervosa (BN) - e ao conceito de alimentação saudável, numa abordagem biopsicosocial.

\section{M É T O D O S}

A fim de realizar uma revisão da literatura, procedeu-se à pesquisa da totalidade de artigos científicos, publicados desde 1997, nos bancos de dados MedLine, PubMed, Lilacs e SciELO. A palavra-chave selecionada para a pesquisa foi "orthorexia nervosa" e a busca foi realizada nos seguintes campos: título, resumo e descritores. As listas de referências de cada artigo foram analisadas para encontrar publicações adicionais. Todas as referências encontradas foram utilizadas (exceto dois artigos suecos e um artigo alemão com texto e resumo indisponíveis).

A partir dos artigos selecionados, o comportamento alimentar ortoréxico foi descrito e comparado com os comportamentos típicos dos transtornos alimentares. Os estudos sobre ortorexia nervosa foram apresentados numa abordagem descritiva. Os grupos ou populações vulneráveis ao desenvolvimento de ortorexia nervosa apontados pelos estudos foram identificados com vistas a informar os profissionais da área da saúde, em especial nutricionistas, sobre a necessidade de rever o conceito de alimentação saudável e alertá-los acerca da existência desse comportamento e suas possíveis consequências indesejáveis sobre a saúde. Para tanto, o conceito de alimentação saudável foi revisto à luz de questionamentos levantados pelas características do comportamento ortoréxico.

\section{RESULTADOSE DISCUSSÃO}

Conforme se pode observar na Tabela 1, os estudos sobre ortorexia nervosa são escassos até o momento, possivelmente, pelo menos em parte, pelo fato de tal comportamento alimentar ter sido descrito há pouco mais de dez anos ${ }^{8}$ e somente começou a ser investigado de forma sistemática há seis anos, quando foi publicado o primeiro artigo original ${ }^{9}$. Até o momento, são encontradas vinte e uma publicações, sendo apenas nove artigos originais de pesquisa ${ }^{9-17}$. Os demais trabalhos consistem de: três artigos sobre a descrição e caracterização da ortorexia nervosa ${ }^{18-20}$, dois estudos de casos clínicos ${ }^{21,22}$ e duas cartas ao editor ${ }^{23,24}$. Cinco trabalhos estão publicados em periódicos de difícil acesso ${ }^{25-29}$, o que inviabilizou a apreciação dos mesmos. Todos os artigos

Tabela 1. Características dos artigos sobre ortorexia nervosa publicados na literatura científica indexada.

\begin{tabular}{clllll}
\hline Referência & Local & Ano & Idioma & Tipo de artigo & Disponibilidade online \\
\hline 20 & Inglaterra & 2001 & Inglês & Caracterização da ON & Sim \\
21 & Estados Unidos & 2004 & Inglês & Caso clínico & Sim \\
9 & Itália & 2004 & Inglês & Artigo original & Não \\
22 & Espanha & 2005 & Inglês & Caso clínico & Sim \\
11 & Itália & 2005 & Inglês & Artigo original & Sim \\
18 & Estados Unidos & 2005 & Inglês & Caracterização da ON & Sim \\
10 & Áustria & 2006 & Inglês & Artigo original publicado na seção "Carta ao editor" & Sim \\
19 & Espanha & 2007 & Espanhol & Caracterização da ON & Sim \\
12 & Turquia & 2007 & Inglês & Artigo original & Sim \\
13 & Suécia & 2008 & Inglês & Artigo original & Sim \\
23 & Brasil & 2008 & Inglês & Carta ao editor & Sim \\
24 & Suécia & 2008 & Inglês & Carta ao editor (resposta) & Sim \\
14 & Turquia & 2008 & Inglês & Artigo original & Sim \\
15 & Turquia & 2009 & Inglês & Artigo original & Sim \\
16 & Turquia & 2010 & Inglês & Artigo original & Sim \\
17 & Alemanha & 2010 & Inglês & Artigo original & Sim \\
\hline
\end{tabular}

ON: ortorexia nervosa. 
originais sobre ortorexia nervosa foram realizados na Europa, sendo quatro destes na Turquia12,14-16, dois na Itália ${ }^{9,11}$, um na Áustria ${ }^{10}$, um na Suécia ${ }^{13}$ e um na Alemanha17.

\section{Ortorexia nervosa: características gerais}

O termo ortorexia nervosa foi criado por Steven Bratman, médico americano, que sugeriu esse quadro ou condição como um novo comportamento alimentar transtornado ${ }^{8}$. A ortorexia nervosa não se encontra definida no manual de diagnósticos de TA da APA, DSM-IV7 , ou mesmo no manual de diagnósticos da OMS, CID-10 30 . Tampouco há um consenso sobre a ortorexia nervosa poder vir a ser considerada um novo TA 23,24 .

Bratman ${ }^{8,31}$ descreveu a ortorexia nervosa como uma fixação pela saúde alimentar caracterizada por uma obsessão doentia com o alimento biologicamente puro, acarretando restrições alimentares significativas. Trata-se de indivíduos com escolhas alimentares acompanhadas de uma preocupação exagerada com a qualidade dos alimentos, a pureza da dieta (livre de herbicidas, pesticidas e outras substâncias artificiais) e o uso exclusivo de "alimentos politicamente corretos e saudáveis" 8,10,11,18.

Para Bratman ${ }^{31}$, o objetivo dos indivíduos com comportamento ortoréxico é ingerir alimentos que contribuam para o bom funcionamento do organismo e "libertem o corpo e a mente de impurezas" a fim de alcançarem um corpo saudável e maior qualidade de vida. O autor também associou esse comportamento com o desprezo pelos que não seguem os mesmos "padrões elevados" de alimentação, a obsessão, a solidão, o evitar a prática social das refeições, a insatisfação com a própria condição e a autoimposição para tentar esclarecer outros acerca dos alimentos "saudáveis".

Ao propor, nomear e caracterizar a ortorexia nervosa, Bratman ${ }^{31}$ também levou em conta a avaliação de si próprio quando, em certa fase da vida, apresentava-se excessivamente preocu- pado com certos aspectos alimentares: a qualidade e o frescor dos vegetais, a ponto de cultivá-los para uso próprio; a contagem da mastigação a cada colherada de alimento; o local de alimentação e a quantidade de comida. De acordo com seu testemunho pessoal:

Era um vegetariano, comia legumes frescos e de qualidade plantados por mim, mastigava cada colherada mais de 50 vezes, comia sempre sozinho, em local sossegado, e deixava o meu estômago parcialmente vazio, no final de cada refeição. Tornei-me um presunçoso que desdenhava qualquer fruto colhido da árvore há mais de quinze minutos. Durante um ano fiz esta dieta, senti-me forte e saudável. Observava com desprezo àqueles que comiam batatas fritas e chocolates como meros animais reduzidos à satisfação dos seus desejos. Mas não estava satisfeito com a minha virtude e sentia-me sozinho e obcecado. Evitava a prática social das refeições e obrigava-me a esclarecer familiares e amigos acerca dos alimentos.

Recentemente, Bartrina ${ }^{19}$ complementou a caracterização da ortorexia nervosa, propondo outros sintomas, tais como: evitação extrema de corantes, aromatizantes, conservantes, pesticidas, alimentos geneticamente modificados, alimentos com muito sal ou muito açúcar e até usar utensílios de cozinha de modo ritualístico (p.ex.: somente cerâmica ou madeira). Além disso, a autora descreve o perfil dos indivíduos vulneráveis à ortorexia nervosa: "pessoas meticulosas, organizadas e com exacerbada necessidade de autocuidado ou proteção". Esse grupo inclui mulheres, adolescentes, pessoas adeptas de modismos alimentares e de hábitos alimentares alternativos, como vegetarianismo e dieta macrobiótica e também atletas que se dedicam a esportes como fisiculturismo e atletismo.

Segundo Donini et al. ${ }^{11}$, a ortorexia nervosa se aproximaria de um distúrbio de personalidade ou de comportamento (do ponto de vista 
psiquiátrico), podendo ter relações com as crenças ou as atitudes ligadas a costumes religiosos ou filosóficos, já que muitas religiões valorizam práticas alimentares ascéticas ${ }^{32}$. O comportamento ortoréxico também poderia começar de modo inocente, com o desejo de curar ou prevenir doenças crônicas e melhorar o estado de saúde. O comportamento requer considerável autodisciplina e autocontrole para seguir a dieta, que difere radicalmente dos hábitos alimentares adquiridos na infância, do estilo de vida da sociedade e da cultura que o rodeia. A ideologia seguida faz com que, na procura por um corpo saudável, elimine-se tudo o que é "nefasto" ou "artificial", e a alimentação passe a ser a preocupação central do dia a dia $^{31}$.

A presença de um componente econômico é apontada como um fator limitante do quadro ortoréxico. Assim, um indivíduo que não tem poder aquisitivo não poderia condescender com uma obsessão por alimentos onerosos como muitos dos que seriam classificados como saudáveis (ex.: alimentos orgânicos) ${ }^{18}$.

Na busca da "pureza alimentar", os indivíduos com comportamento ortoréxico podem tornar-se muito seletivos em relação aos alimentos que escolhem. Dessa forma, acabam optando por condutas alimentares cada vez mais restritivas que podem levar à carência de determinados nutrientes, colocando em risco a própria saúde. Com o tempo, passam a dedicar cada vez mais tempo para planejar, comprar, preparar e confeccionar os alimentos que vão consumir. Desse modo, despendem muito tempo e esforço em torno do ato de comer (conhecido como a "espiritualidade da cozinha") $)^{31}$.

Outra consequência seria o afastamento da sociedade, porque os indivíduos com ortorexia nervosa se sentem na obrigação de esclarecer, elucidar e convencer familiares e amigos acerca dos prejuízos à saúde que, sob sua perspectiva, seriam causados pelos produtos processados, pesticidas e fertilizantes artificiais. Esse comportamento pode gerar conflitos e dificuldades de relacionamento, de modo a levar o indivíduo ao isolamento social na sua luta por uma condição alimentar perfeccionista ${ }^{31}$. Alguns autores ${ }^{12,16}$ discutem ainda se a mídia e a propaganda exercem alguma influência na etiologia da ortorexia nervosa e na promoção do comportamento ortoréxico.

O Quadro 1 resume as principais características da ortorexia nervosa a partir das descrições e discussões levantadas nos artigos revisados. Entretanto, vale ressaltar que o próprio conceito de ortorexia nervosa tem sido alvo de discussão nos meios científicos ${ }^{23,24}$, o que impossibilita o detalhamento do quadro de modo completamente claro e objetivo.

\section{Ortorexia nervosa e transtornos alimentares: uma análise comparativa}

O comportamento ortoréxico apresenta similaridades e diferenças quando comparado com os comportamentos alimentares característicos dos TA. À semelhança das pessoas com TA, aquelas com ortorexia nervosa também demonstram sentimentos, crenças, pensamentos e comportamentos para com os alimentos que podem ser considerados obsessivos, extremistas e restritivos. Entre eles estão: ansiedade, necessidade de controle e de seguir regras rígidas, desejo de sentir-se puro e tendência ao perfeccionismo transferidos para o ato de comer ${ }^{33}$. Sobre a questão da pureza e ascetismo, é interessante observar historicamente os relatos de santas e beatas da igreja católica descritas como as antigas "anoréxicas" - anorexia santa -, que eram motivadas pela pureza de espírito e caráter, abnegação e negação dos prazeres terrenos e carnais ${ }^{34}$. Tanto os TA quanto a ortorexia nervosa parecem ser impulsionados por pressões sociais, sendo o primeiro grupo afetado pelos padrões estéticos de beleza e pelo culto ao corpo, enquanto o segundo grupo seria influenciado pela ênfase no viver saudável - novamente em seu foco biológico ${ }^{18}$.

Fazendo uma analogia entre a ortorexia nervosa e os TA, pode-se dizer que a visão sobre alimentação saudável entre os pacientes com TA 
350 | M.C.T. MARTINS et al.

Quadro 1. Características principais da ortorexia nervosa.

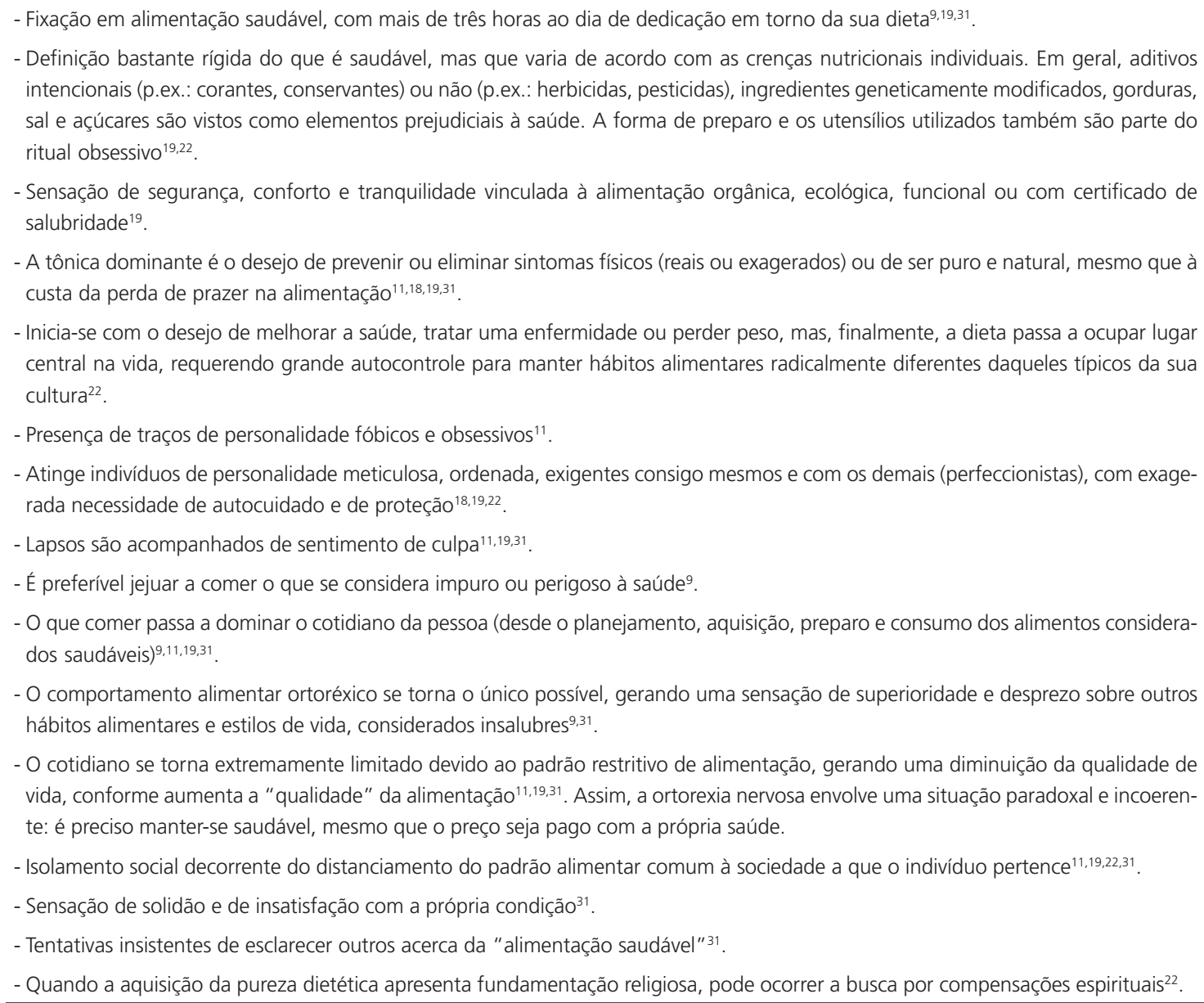

tende a ser ortoréxica, uma vez que apresentam uma concepção perfeccionista do que seja alimentação saudável, com uma série de crenças e mitos sobre nutrição e alimentação ${ }^{35}$. As crenças podem tornar-se normas rígidas e obrigações íntimas. Essas regras ou crenças contêm em sua formulação expressões como "deve-se", "tem-se que", isto é, imposições que limitam ou impedem a pessoa de quebrá-las. A pessoa sujeita a crenças tão rígidas perde o poder de escolha, torna-se cronicamente ansiosa, frustrada e desapontada consigo, uma vez que não consegue cumprir todas as normas impostas para atingir o ideal alcançado.

Algumas características ideológicas e comportamentais, no entanto, distinguem os pacientes com TA dos indivíduos com ortorexia nervosa.
Pacientes com AN e BN estão motivados pela perda de peso ${ }^{33}$, enquanto indivíduos com ortorexia nervosa são orientados pelo objetivo de alcançar a "dieta saudável perfeita". Ademais, os primeiros raramente formam comunidades (com a exceção dos controversos web sites pró AN e pró BN), enquanto os indivíduos obcecados por uma dieta perfeita procuram formar grupos com a mesma ideologia ${ }^{19}$.

Outro aspecto distintivo pode ser a receptividade dos indivíduos ao tratamento. Sugere-se que a resistência ao tratamento possa ser menor entre os indivíduos com ortorexia nervosa em relação aos indivíduos com TA, uma vez que os primeiros estão preocupados com a própria saúde ${ }^{18}$, enquanto os pacientes com TA tendem à negação do distúrbio ou da gravidade de seus quadros ${ }^{33}$. 
No entanto, uma dificuldade pode ser convencer os indivíduos com ortorexia nervosa de que seu comportamento é inadequado ou mesmo insalubre, já que eles se consideram saudáveis e disciplinados e podem não imaginar nenhum tipo de consequência adversa de seu comportamento alimentar. O tratamento da ortorexia nervosa parece requerer conduta semelhante à adotada para os TA no que diz respeito à necessidade de uma abordagem interdisciplinar que envolva médicos, psicólogos ou psicoterapeutas e nutricionistas $^{19}$.

\section{Estudos sobre ortorexia nervosa}

A compreensão do comportamento ortoréxico e a identificação dos grupos vulneráveis à ortorexia nervosa têm sido dificultadas por diversos fatores. O número de estudos realizados até o momento ainda é pequeno, sendo a totalidade deles do tipo transversal. Os trabalhos existentes foram realizados em localidades diversificadas (Alemanha, Áustria, Itália, Suécia e Turquia), de modo que sua tradução para outros contextos socioculturais fica limitada, impossibilitando a universalização dos resultados. Também não há uniformidade dos instrumentos ou dos critérios de classificação empregados para identificação da ortorexia nervosa, e os instrumentos ainda carecem de melhor validação. Além disso, não se pode descartar a interferência de variações decorrentes dos métodos de tradução dos instrumentos, que nem sempre são descritos ou utilizam técnicas apropriadas.

Apesar das limitações dos estudos sobre a ortorexia nervosa, os trabalhos trazem informações que podem ser consideradas relevantes como ponto de partida para entendimento do comportamento ortoréxico. A seguir, os estudos são apresentados numa análise descritiva, partindo dos instrumentos desenvolvidos em direção aos grupos vulneráveis apontados na literatura científica.

Ao descrever inicialmente o comportamento ortoréxico, Bratman ${ }^{8}$ desenvolveu um teste, envolvendo atitudes em relação aos alimentos
(BOT ou Bratman's orthorexia test), com dez afirmativas que permitem respostas dicotômicas: "sim" ou "não". Por exemplo: "Sinto-me culpado se me desvio de meus hábitos dietéticos", com um escore de 0-10, com cada "sim" correspondendo a um ponto. Até o momento, três trabalhos empregaram o BOT, no entanto não houve uniformidade entre eles quanto ao critério de classificação para identificação do quadro ortoxéxico ${ }^{10,12}$. Kinzl et al. ${ }^{10}$ consideraram escores entre 2 e 3 indicativos de sinais de ortorexia e a partir de 4 como ortorexia nervosa. Empregando uma versão do BOT traduzida para o sueco por Rössner ${ }^{27}$. Eriksson et al. ${ }^{13}$ consideraram que se o respondente somasse menos que 5 pontos, sua atitude em relação aos alimentos era considerada saudável; entre 5 e 9, sua atitude alimentar era considerada "fanática por saúde", e somando 10 pontos (ao responder "sim" a todas as questões), seu relacionamento com os alimentos era considerado obsessivo, caracterizando a ortorexia nervosa. Korinth et al. ${ }^{17}$ utilizaram a versão alemã do BOT proposta por Kinzl et al..$^{29}$ e computaram o escore obtido pela somatória das respostas positivas para as dez questões. Os autores compararam estatisticamente os escores médios obtidos entre os grupos estudados, mas não calcularam a frequência da ortorexia nervosa. Até o momento o BOT não foi submetido à avaliação psicométrica.

Pesquisadores italianos publicaram o primeiro estudo preliminar para identificação da ortorexia nervosa e tentativa de mensuração da sua dimensão após a observação das características comportamentais de 28 pessoas que exibiam sintomas do quadro, segundo os autores ${ }^{9}$. O mesmo grupo desenvolveu e validou um instrumento para diagnóstico da ortorexia nervosa, denominado ORTO-1511, contendo 15 questões de múltipla escolha (sempre, frequentemente, às vezes e nunca) que inclui alguns dos itens do BOT e agrega outros criados. As questões do ORTO-15 abordam atitudes obsessivas dos indivíduos com ortorexia nervosa quanto à escolha, compra, preparo e consumo de alimentos considerados saudáveis. O teste mostrou boa capacidade 
preditiva em um ponto de corte de 40 (com $73,8 \%$ de eficácia, $55,6 \%$ de sensibilidade e $75,8 \%$ de especificidade), verificados com uma amostra-controle. Entretanto, os próprios autores ressaltam que o teste não identifica comportamentos obsessivo-compulsivos e recomendam que novas questões deveriam ser acrescentadas e novas avaliações do instrumento realizadas.

O ORTO-15 foi traduzido para o turco e empregado para estudar a prevalência da ortorexia nervosa em médicos residentes ${ }^{12}$ e em artistas $^{15}$ turcos. Outro grupo de pesquisadores tur$\cos ^{14}$ avaliou as propriedades psicométricas da versão turca do ORTO-15, encontrando uma baixa consistência interna (Alpha de Cronbach $=0,44$ ). Esses autores modificaram o instrumento pela remoção de quatro questões cujos pesos eram menores do que 0,50 , chegando a uma nova versão denominada ORTO-11, que obteve Alpha de Cronbach de 0,62 e que já foi empregada em dois trabalhos: um deles investigou a relação entre ortorexia nervosa, algumas variáveis demográficas e sintomas de atitude alimentar e de transtorno obsessivo compulsivo ${ }^{14}$; o outro empregou o ORTO-11 para estudar a prevalência de ortorexia nervosa entre estudantes de medicina turcos ${ }^{16}$.

Na tentativa de identificar grupos vulneráveis à ortorexia nervosa, três estudos sugerem que estudantes e profissionais da área de saúde, tais como estudantes de medicina ${ }^{16}$, nutricionistas ${ }^{10}$ e médicos ${ }^{1}$, podem ter maior predisposição a comportamentos ortoréxicos. Em contrapartida, um estudo recente questiona essa proposição ${ }^{17} \mathrm{em}$ relação aos estudantes de nutrição.

No trabalho de Kinzl et al. ${ }^{10}$, realizado com 283 nutricionistas austríacas do sexo feminino, sugeriu-se que a ortorexia nervosa avaliada pelo BOT parece ser um comportamento frequente nessas profissionais, uma vez que $34,9 \%$ das participantes do estudo apresentaram algum comportamento ortoréxico e 12,8\% apresentaram ortorexia nervosa. O comportamento ortoréxico das nutricionistas foi assim caracterizado: ganho de autoestima ao comer alimentos saudáveis (8,8\%), sensação de culpa por desviar-se da dieta $(4,6 \%)$, evitação de comer fora de casa por temer uma alimentação não saudável (2,5\%), evitação de comer com outras pessoas $(2,5 \%)$, e levar os próprios alimentos quando tiver que comer fora de casa $(1,1 \%)$. Usando o mesmo instrumento no mesmo idioma (alemão), recentemente Karinth et al. ${ }^{17}$ avaliaram a tendência para ortorexia nervosa em estudantes de nutrição de universidades alemãs. Foram comparados alunos do primeiro ano do curso ( $n=123)$ e dos semestres mais avançados ( $n=96$ ) com um grupo-controle de estudantes de outros cursos ( $n=68$ e $n=46$ ), respectivamente. Embora os estudantes de nutrição apresentassem maiores níveis de restrições dietéticas, eles não demonstraram maior tendência para ortorexia nervosa, já que não houve diferença entre os escores do BOT obtidos pelos alunos de nutrição em relação ao grupo-controle. Além disso, a tendência para ortorexia nervosa foi decrescente entre os estudantes de nutrição mais avançados, que também apresentaram escolhas alimentares mais saudáveis. É importante salientar, entretanto, que as limitações desse trabalho impedem que suas observações sejam generalizadas para diferentes países e contextos culturais, por tratar-se de um estudo transversal realizado com uma pequena amostra exclusivamente de estudantes alemães.

Dois trabalhos realizados na Turquia sugerem que médicos residentes e estudantes de medicina podem constituir grupos com comportamento altamente sensível em relação à ortorexia nervosa. No estudo de Bagci Bosi et al. ${ }^{12}$, realizado com um grupo de 318 médicos de ambos os gêneros (169 homens e 149 mulheres), residentes na faculdade de medicina de Ancara, o comportamento ortoréxico avaliado pelo ORTO-15 foi observado em $45,5 \%$ dos participantes. Mais recentemente, num outro estudo transversal, utilizando o instrumento ORTO-11 entre 878 estudantes de medicina turcos de ambos os sexos (464 homens e 359 mulheres) ${ }^{16}$, também se encontrou uma alta ocorrência de ortorexia nervosa $(43,6 \%)$. A tendência foi significativamente mais alta entre estudantes do sexo masculino. Fidan et al. ${ }^{16}$ obser- 
varam ainda maior ocorrência para ortorexia nervosa entre estudantes mais jovens do sexo masculino. No mesmo estudo, baseado nos escores do EAT-40 (Eating Attitude Test), os estudantes com comportamento de risco para TA também apresentaram tendência para ortorexia nervosa.

Outros três estudos foram realizados em grupos distintos e trazem informações sobre o perfil dos indivíduos com tendência ao desenvolvimento do comportamento ortoréxico (pessoas com sintomas de ansiedade, obsessivo-compulsivas e aquelas que supervalorizam o corpo perfeito).

Eriksson et al. ${ }^{13}$ utilizaram o BOT em 251 praticantes de atividade física suecos de ambos os gêneros (85 homens e 166 mulheres) para avaliar o impacto de dois fatores sobre o teste de ortorexia nervosa: (a) ansiedade psicossocial e (b) atitudes socioculturais relacionadas com a aparência física. Os participantes que obtiveram maiores escores no questionário de atitudes socioculturais relacionadas com a aparência física (SATAQ) no subdomínio internalização também tiveram altos escores no BOT. A internalização indica a adoção e/ou aceitação dos ideais sociais de magreza (para as mulheres) e de muscularidade (para os homens). Observou-se também que as mulheres com maior frequência de exercícios físicos e com maiores escores na escala de ansiedade psicossocial (SPAS) apresentaram escores mais altos no BOT. Esse trabalho evidencia que as crenças e comportamentos ortoréxicos também podem estar relacionados com a supervalorização e a busca do corpo perfeito presente na sociedade moderna. De fato, Mathiew ${ }^{18}$ e Bratman ${ }^{31}$ e anteriormente sugeriram que adolescentes esportistas do sexo feminino dedicadas ao fisiculturismo e atletismo seriam possíveis grupos vulneráveis à ortorexia nervosa.

No maior estudo realizado até o momento sobre a ortorexia nervosa, Arusoglu et al..$^{14}$ investigaram 994 voluntários da área acadêmica e administrativa da Universidade de Hacettepe, Turquia, com idade entre 19 e 66 anos. Empregando o instrumento ORTO-11, encontraram uma relação positiva entre comportamento de risco para TA (avaliado pelo EAT-40), sintomas obsessivo-compulsivos e os sintomas de ortorexia nervosa. Nesse estudo, as mulheres exibiram mais sintomas ortoréxicos do que os homens.

Ainda identificando ortorexia nervosa em outros grupos, Aksoydan \& Camci'15 avaliaram 94 artistas turcos de ambos os gêneros (39 homens e 55 mulheres) com idade média de 33 anos e encontraram uma ocorrência de 56,45\% dos indivíduos com sintomas de ortorexia nervosa, sendo esses valores significativamente diferentes entre cantores de ópera $(81,8 \%)$, músicos da orquestra $(36,4 \%)$ e dançarinos de balé $(32,1 \%)$. Não houve correlação entre os escores do ORTO15 e características como gênero, idade, escolaridade, experiência de trabalho, índice de massa corpórea, tabagismo ou consumo de álcool. O grupo estudado possui grau de escolaridade e nível socioeconômico superiores aos encontrados na população turca em geral. Os autores discutem que, além disso, naquele país, os artistas são considerados modelos de aparência física e de estilo de vida, o que seriam possíveis fatores contribuintes para a alta ocorrência de ortorexia nervosa nessa população.

\section{Ortorexia nervosa: uma oportunidade para repensar o conceito de alimentação saudável}

A discussão sobre ortorexia nervosa é interessante para se pensar sobre o conceito de alimentação saudável com um enfoque biopsicossocial.

Tradicionalmente, o conceito de "alimentação saudável" tem sido relacionado apenas com o foco biológico e na adequação nutricional resultante de uma alimentação variada. Para Fisberg et al. ${ }^{36}$, "mediante uma alimentação variada em quantidades adequadas, pode-se obter uma dieta equilibrada, ou seja, a que proporciona os nutrientes necessários para atender às necessidades do organismo". 
O ser humano, no entanto, não tem necessidades apenas biológicas, por isso é preciso que se amplie a definição de alimentação saudável além do biológico. Dessa forma, o comportamento alimentar deve envolver todas as formas de convívio com o alimento. Entendido como um processo, o comportamento alimentar constitui um conjunto de ações realizadas com relação ao alimento, que têm início no momento da decisão e envolvem a disponibilidade, o modo de preparar, os utensílios usados, as características, os horários e a divisão da alimentação nas refeições do dia, as preferências e aversões alimentares e finalmente encerra com o alimento sendo ingerido ${ }^{37}$.

O comportamento está inserido em uma definição ainda mais complexa, que é a atitude alimentar. Alvarenga et al. ${ }^{38}$ definiram atitude alimentar como crenças, pensamentos, sentimentos, comportamentos e relacionamento para com os alimentos. Esta visão engloba, portanto, as cognições e os conhecimentos que o indivíduo tem sobre alimentação e a carga afetiva em relação aos alimentos. Estes fatores predispõem a determinadas ações que marcam o relacionamento de uma pessoa com sua alimentação.

Obviamente a atitude alimentar não é definida por parâmetros biológicos e o ambiente socioemocional de um indivíduo tem papel fundamental em relação a suas atitudes. É possível imaginar a existência de múltiplos padrões de atitude alimentar com variáveis graus de distanciamento (ou aproximação) de uma situação saudável. Uma atitude alimentar saudável em relação ao alimento envolveria, portanto, um entendimento do seu papel fisiológico, emocional e social, e não apenas a ausência de comportamentos alimentares inadequados (como os presentes nos TA).

Quando as atitudes alimentares são levadas em consideração, o alimento deixa de fazer parte apenas da esfera fisiológica, e as pessoas não baseiam suas escolhas alimentares somente no valor nutricional ou na contribuição energética dos alimentos. Assim, uma pessoa cujo consumo alimentar alcance todas as suas necessidades nutricionais pode não ter uma atitude alimentar adequada - caso da ortorexia nervosa -, e essa alimentação não poderia ser então considerada verdadeiramente saudável.

Tais considerações levantam alguns questionamentos acerca da visão do nutricionista sobre alimentação saudável, fruto de uma formação acadêmica excessiva e/ou até mesmo exclusivamente baseada na qualidade nutritiva da dieta. Esse tipo de visão poderia gerar crenças e comportamentos ortoréxicos, que por sua vez seriam transmitidos aos seus pacientes e clientes, em um efeito do tipo dominó.

Beumont et al. ${ }^{3}$ ressaltam que "o comer adequadamente não está relacionado apenas com a manutenção da saúde, mas também com um comportamento socialmente aceitável, flexibilidade e satisfação". Assim, entende-se que o conceito de alimentação saudável deva ser explorado em toda sua amplitude.

As necessidades e os recursos que envolvem a alimentação englobam todo o campo culinário e gastronômico, inclusive as culturas populares, as influências religiosas, a biologia e a medicina. Também afetam e são afetados por oscilações econômicas, acontecimentos políticos, tensões sociais, condições meteorológicas, medidas fiscais e fenômenos sanitários. Por esse motivo, sabe-se que dentre outros assuntos relacionados a uma vida saudável, talvez o mais complexo de definir, nas atuais circunstâncias, seja de fato a alimentação. Desse modo, o conceito de alimentação saudável pode diferir quando se consideram culturas diferentes, ainda que seja construído com os mesmos princípios básicos. Por esse motivo, é importante conhecer os fatores geográficos, econômicos, sociais e psicológicos que juntos determinarão as escolhas alimentares em grupos distintos e específicos ${ }^{37}$.

Num exercício de assumir a alimentação como o resultado de múltiplas interações entre o biológico e o sociocultural, buscando também a humanização da abordagem sobre alimentação saudável, alguns autores têm alertado sobre o perigo de que os profissionais de saúde enxer- 
guem o corpo apenas como "domicílio dos nutrientes" que "dão sentido à orientação sobre uma alimentação saudável" e refutem outras dimensões, "coibindo valores simbólicos inscritos na alimentação cotidiana" 39 . Os autores entendem que "as condições alimentares são decorrentes de um sistema complexo das relações sociais e que os fatores biológicos estão incessantemente comungados aos efeitos das produções da realidade social" . Poulain \& Proença ${ }^{40}$ propõem o conceito de "espaço social alimentar" como "um instrumento de estudo dos modelos alimentares, assinalando a conexão bioantropológica de um grupamento humano ao seu meio. Neste conceito destacam-se seis dimensões principais que focalizam espaços e sistemas diversos: o comestível, a produção alimentar, o culinário, os hábitos de consumo alimentar, a temporalidade e as diferenciações sociais". Assim, Poulain \& Proença ${ }^{40}$ consideram um modelo alimentar como sendo "a configuração particular do espaço social alimentar".

Ao percorrer o trilho dessa discussão sobre alimentação saudável, observa-se uma linha multifacetária que engloba aspectos culturais, sociais, econômicos, nutricionais, psicológicos, ambientais, religiosos e tecnológicos entre outros. Dessa forma, parece evidente que os comportamentos alimentares saudáveis devem apresentar certo equilíbrio harmônico entre os diversos aspectos envolvidos com a alimentação humana.

\section{O N CLUS Ã O}

Ainda há um longo caminho de investigação científica a ser percorrido para que a ortorexia nervosa seja amplamente conhecida e compreendida. São necessários mais estudos para descrever de modo mais completo o comportamento ortoréxico, sua etiologia, possível diagnóstico, tratamento, grupos e/ou populações vulneráveis. Embora a ortorexia nervosa ainda não seja oficialmente reconhecida como um TA, estudos sobre esse comportamento alimentar poderão embasar o seu possível futuro reconhecimento. Ademais, trabalhos sobre esse tema poderão fornecer aos profissionais da área de saúde informações necessárias para que possam identificar o indivíduo com comportamento ortoréxico e encaminhá-lo ou tratá-lo adequadamente.

O desenvolvimento do presente trabalho levantou uma temática nova e pouco discutida em nutrição. Destaca-se a importância de que profissionais da área da saúde estejam atentos e atualizados sobre o ato de comer e suas implicações. O conceito e a adoção de hábitos saudáveis e seguros na alimentação devem estar afastados de atitudes e práticas obsessivas e perfeccionistas, ainda que elas sejam motivadas por um desejo de alcançar a pureza da dieta a qualquer custo.

A prevalência da ortorexia nervosa ainda é desconhecida em nossa sociedade. Os trabalhos disponíveis até o momento foram realizados em lugares muito diversos (Alemanha, Áustria, Itália, Suécia e Turquia), em amostras pequenas e específicas, de modo que a tradução para nossa realidade deve ser feita de forma muito cuidadosa, visto não ser possível uma universalização dos resultados. Entretanto, apesar das possíveis limitações metodológicas e da necessidade de maior definição conceitual presente nos poucos estudos realizados até o momento sobre a ortorexia nervosa, os resultados apontam para algumas tendências em certos grupos, como profissionais da área de saúde - por exemplo, médicos e nutricionistas. Assim, talvez seja apropriado iniciar estudos em nosso meio, fazendo um levantamento desse comportamento alimentar alterado em populações aparentemente mais vulneráveis. A investigação da prevalência da ortorexia nervosa em tais populações, acompanhada de esclarecimentos e orientações adequadas, pode ser instrumento útil para que esses profissionais encontrem o devido equilíbrio em suas práticas alimentares pessoais e evitem disseminar orientações rígidas sobre alimentação saudável em sua prática profissional.

\section{OLABORADORES}

M.C.T. MARTINS concebeu e desenvolveu a ideia básica para o presente estudo, conduziu a elabo- 
356 | M.C.T. MARTINS et al.

ração do projeto de pesquisa, efetuou a busca bibliográfica, participou da discussão e da interpretação dos resultados e preparou a redação final do artigo. M.S. ALVARENGA e F.B. SCAGLIUSI participaram da elaboração do projeto de pesquisa, da busca bibliográfica, da discussão e interpretação dos resultados e da elaboração do artigo final. S.V.A. VARGAS e K.S.C.J. SATO participaram da elaboração do projeto de pesquisa, da busca bibliográfica e da redação inicial do artigo.

\section{REFERÊ NCIAS}

1. Sichieri R, Coitinho DC, Monteiro JB, Coutinho WF. Recomendações de alimentação e nutrição saudável para a população brasileira. Arq Bras Endocrinol Metab. 2000; 44(3):227-32. doi: 10.1590/S0004-2 7302000000300007

2. Rozin $\mathrm{P}$, Ashmore M, Markwith M. Lay american conceptions of nutrition: dose insensitivity, categorical thinking, contagion, and the monotonic mind. Health Psychol. 1996; 15(6):438-47.

3. Beumont PJV, O'Connor M, Lennerts $W$, Touyz W. Nutritional counseling in the treatment of bulimia. In: Fichter MM. Bulimia nervosa: basic research, diagnosis and therapy. London: John Wiley \& Sons; 1990.

4. Sizer FS, Whitney EM. Nutrição conceitos e controvérsias. $8^{a}$ ed. Barueri: Manole; 2003. p.147-59.

5. American Dietetic Association. Position of the American Dietetic Association: total diet approach to communicating food and nutrition. J Am Diet Assoc. 2007; 107(7):1224-32.

6. American Dietetic Association. Position of the American Dietetic Association: nutrition intervention in the treatment of anorexia nervosa, bulimia nervosa, and other eating disorders. J Am Diet Assoc. 2006; 106(12):2073-82.

7. American Psychiatry Association. Diagnostic and statistical manual of mental disorders (DSM-IV). $4^{\text {th }}$ ed. Washington (DC): APA; 1994.

8. Bratman SMD. Original essay on orthorexia, 1997. [cited 2009 Jun 25]. Available from: <http//www. orthorexia.com>.

9. Donini LM, Marsili D, Graziani MP, Imbriale M, Canella C. Orthorexia nervosa: a preliminary study with a proposal for diagnosis and an attempt to measure the dimension of the phenomenon. Eat Weight Disord. 2004; 9(2):151-7.

10. Kinzl JF, Hauer K, Traweger C, Kiefer I. Orthorexia nervosa in dieticians. Psychother Psychosom. 2006; 75(6):395-6.
11. Donini LM, Marsili D, Graziani MP, Imbriale M Carnella C. Orthorexia nervosa: validation of a diagnosis questionnaire. Eat Weight Disord. 2005; 10(2):e28-e32.

12. Bagci Bosi AT, Çamur D, Güler C. Prevalence of orthorexia nervosa in resident medical doctors in the faculty of medicine (Ankara, Turkey). Appetite. 2007; 49(3):661-6.

13. Eriksson L, Baigi A, Marklund B, Lindgren EC. Social physique anxiety and sociocultural attitudes toward appearance impact on orthorexia test in fitness participants. Scand J Med Sci Sports. 2008; 18(3): 389-94

14. Arusoglu G, Kabakçi E, Köksal G, Kutluay Merdol T. Orthorexia nervosa and adaptation of ORTO-11 into Turkish. Turk J Psychiatr. 2008; 19(3):1-9.

15. Aksoydan E, Camci N. Prevalence of orthorexia nervosa among Turkish performance artists. Eat Weight Disord. 2009; 14(1):33-7.

16. Fidan T, Ertekin V, Isikay $S$, Kirpinar I. Prevalence of orthorexia among medical students in Erzurum, Turkey. Compr Psychiatry. 2010; 51(1):49-54.

17. Karinth A, Schiess S, Westenhoefer J. Eating behaviour and eating disorders in students of nutrition sciences. Public Health Nutr. 2010; 13(1): 32-7.

18. Mathieu J. What is orthorexia? J Am Diet Assoc. 2005; 105(10):1510-12.

19. Bartrina JA. Ortorexia o la obsesión por la dieta saludable. Arch Latinoam Nutr. 2007; 57(4):313-5.

20. MacEvilly E. The price of perfection. Nutr Bull. 2001; 26(4):275-6.

21. Cartwright MM. Eating disorder emergencies: understanding the medical complexities of the hospitalized eating disordered patient. Crit Care Nurs Clin North Am. 2004; 16(4):515-30.

22. Catalina Zamora CML, Bote Bonaechea B, Garcia Sánchez F, Rios Rial B. Orthorexia nervosa. A new eating behavior disorder? Actas Esp Psiquiatr. 2005; 33(1):66-8

23. Kummer A, Dias MV, Teixeira, AL. On the concept of orthorexia nervosa. Scand. J Med Sci Sports. 2008; 18(3):395-6.

24. Eriksson L, Baigi A, Marklund B, Lindgren EC. On the concept of orthorexia nervosa: a rebuttal. Scand J Med Sci Sports. 2008; 18(3):397.

25. Nyman $\mathrm{H}$. A direct question: is orthorexia a correct word for a wrong concept? Lakartidningen. 2002; 99(5):423-4.

26. Mader U. Orthorexia: the addiction to nourishing oneself "healthfully". Dtsch Med Wochenschr. 2004; 129(14):728. 
27. Rossner S. Ortorexia nervosa: a new disease? Lakartidningen. 2004; 101(37):2835.

28. Calañas Continente AJ. Ortorexia: um transtorno nutricional de los países desarollados. Rev Nutr Pract. 2003; 7:47-50.

29. Kinzl JF, Hauer K, Traweger C, Kiefer I. Orthorexia nervosa: a frequent eating disorder in dieticians? Ernährungs-Umschau. 2005; 52(11):436-9.

30. Organização Mundial de Saúde. Classificação de transtornos mentais e de comportamento da CID-10. Descrições clínicas e diretrizes diagnósticas. Porto Alegre: Artes Médicas; 1993.

31. Bratman, Orthorexia nervosa: the health food eating disorder. New York: Broadway Books; 2002.

32. Institute of Food Technologists. Proceedings of a symposium on: "religious and philosophical basis of food choices". Food Technol. 1992; 46(10):92-128.

33. American Psychiatry Association. Work Group on Eating Disorders. Practice guidelines for the treatment of patients with eating disorders. $3^{\text {rd }}$ ed. Washington (DC): American Psychiatric Association; 2006.

34. Scagliusi FB, Lourenço BH, Barreto AA, Sato PM, Alvarenga MS, Cordás TA. Modern holy anorexia: how advertising explores and disseminates ascetic ideals. In: Fuchs JN, editor. Eating disorders in adult women. New York: Nova Sciences Publishers; 2008.

35. Alvarenga MS, Dunker KLL. Padrão e comportamento alimentar na anorexia e bulimia nervosa.
In: Philippi ST, Alvarenga MS. Transtornos alimentares: uma visão nutricional. São Paulo: Manole; 2004.

36. Fisberg RM, Villar BS, Colucci ACA, Philippi ST. Alimentação equilibrada na promoção da saúde. In: Cuppari L. Nutrição clínica no adulto. $2^{a}$ ed. Barueri: Manole; 2002.

37. Philippi ST, Alvarenga M. Alimentação saudável: princípios e recomendações. In: Philippi ST, Alvarenga $\mathrm{M}$. Transtornos alimentares: uma visão nutricional. Barueri: Manole; 2004.

38. Alvarenga MS, Scagliusi FB, Philippi ST. Changing attitudes, beliefs and feelings towards food in bulimic patients. Arch Lationoam Nutr. 2008; 58(3): 274-9.

39. Silva DO, Recine EGIG, Queiroz EFO. Concepções de profissionais de saúde da atenção básica sobre a alimentação saudável no Distrito Federal, Brasil. Cad Saúde Públ. 2002; 18(5):1367-77. doi: 10.15 90/S0102-311X2002000500030.

40. Poulain J-P, Proença RPC. O espaço social alimentar: um instrumento para o estudo dos modelos alimentares. Rev Nutr. 2003; 16(3):245-56. doi: 10.1590/S 1415-52732003000300002.

Recebido em: 6/11/2009

Versão final reapresentada em: 26/5/2010 Aprovado em: 3/11/2010 
